\title{
Identification and Research of Adhd and Healthy Controls using Fmri
}

\author{
K.Thaiyalnayaki
}

\begin{abstract}
Analyzing the brain regions for different activations corresponding to the activation input for an experimental setup of task functional MRI or a resting state functional Magnetic Resonance Imaging(fMRI) for a diagnosed or healthy control is a challenging issue as the processing data is voluminous $4 D$ data with nearly 1,51,552 voxels for a single volume of 261 scans fMRI. The data considered for analysis consists of 10 healthy controls and 10 Attention Deficit Hyperactivity Disorder(ADHD) fMRI. The workflow starts with preprocessing the individual scan for realignment, coregistration and Normalisation to Montreal Neurological Institute (MNI) space. Single site scan visit consists of $64 \times 64 x 37$ voxels. Seventy independent components are obtained from processed data by data reduction, Independent Component Analysis (ICA) calculation, Back reconstruction and Component Calibration. ICA performs satisfactorily well on temporal and spatial localization. Visual medial network activation is pronounced in ADHD Controls than in healthy people. Sagittal, Axial and Coronal view of ADHD controls is obtained as component number 42.The analysis is further used for the automatic classification of healthy controls and ADHD people.
\end{abstract}

\section{INTRODUCTION}

\section{INTRODUCTION TO FMRI}

fMRI is a specialized noninvasive neuro imaging technique used for studying human brain function. fMRI is a method for studying the cognitive functions of the brain. Functional imaging potential enhance surgical conclusion and have the prospective to help researchers assess and treat debilitating Central Nervous System disorders[5][10]. ADHD controls find difficulty in sitting motionless, playing silently with less anxiety. fMRI technique for analysing functional localization is concerned to subjects that performed no formulated task ie. resting state. In this study, functional MRI is used as tool to identify automatically the regions of the brain activated for ADHD controls. The task and rest related activation analysis techniques focus on localization of huge datasets [3][4]. A multivariate based ICA is resort to categorize the effects of activation from the statistics.

One of the fMRI scrutiny method most agreed uniquely is signals called sparse kSVD[9]. Independent Component Analysis (ICA) does not assure the autonomy of synchronously occurring salient excited regions in the brain[1]. Alternatively, the sparsity of the signal is more affirmative and data driven fMRI analysis that is obtained entirely depend on the sparsity of the signals is examined. A particular voxel may be considered as a integration of a

Revised Manuscript Received on August 14, 2019.

K.Thaiyalnayaki,Department of ECE, Saveetha School of Engineering, SIMATS, Chennai, Tamilnadu, India (email: thaiyalnayaki@gmail.com) constructed on the sparsity of essential hemodynamic

sparse set of effective components, where individual component has non identical time-series signal patterns [8]. GLM composition for a maximum likelihood estimation of design matrices is another known method for analysis. There is a quest for prolonged independent component analysis of fMRI data to contribute for group inferences and estimating intelligently for various fMRI group studies [4][12]. Independent Component Analysis is used to identify Temporal Independent Components. An estimate of the similarities between unsupervised clustering and ICA is graded by task relevant activation maps, related timecourses, and receiver operating characteristic reasoning. The ICA methods show to extract features comparably well for limited number of independent components however, constrained to the linear mixture theorization

\section{SPM AND ICA}

\section{THEORY OF SPM AND ICA}

ICA in general desires at disintegrating the data matrix, a product of spatial maps and identical time courses where either patterns or time courses are a priori independent. Principal component Analysis is based on uncorrelated and Normality property whereas ICA is in the assumption of statistical Independence. ICA decomposition is represented as

$$
\mathrm{X}=\mathrm{AS}
$$

$\mathrm{X}$, is the matrix of observations. A is mixing matrix which contains co-efficient that mixes the independent components in unknown process. $\mathrm{S}$ is a matrix which contains desired ICs. Both A and S are unknown. Different methods are there to estimate A. Maximum Likelihood estimation is performed and ICs are obtained. In General Linear model fMRI time series is modeled as

$$
\mathrm{Y}=\mathrm{X} \beta+\varepsilon
$$

$\mathrm{Y}$ is a vector of measured data, $\varepsilon$ is error term indicating the difference between the measured data $\mathrm{Y}$ and $\beta, \mathrm{X}$ is design matrix containing regressors.

\section{EXPERIMENTAL ANALYSIS \& RESULTS}

A total of 261 acquisitions of fMRI rest data from open source are considered for analysis. Slice dimension of each acquisition, has $64 \times 64$ matrix with 37 slices and $3.4376 \mathrm{~mm}$ $\mathrm{x} 3.4376 \mathrm{~mm} \times 3.2 \mathrm{~mm}$ in plane resolution, resting state

\section{Published By:}


fMRI scan in a 3T MRI scanner. The changes in signal intensity are performed during spatial realignment, which can arise in the scanning session from intra subject head motion. The images were aligned to the first image of the session in each session. The spatial maps are normalized spatially to a established Talairach space and resampled to respective voxels.

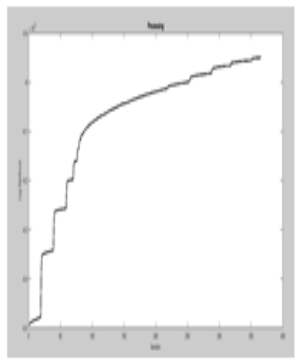

Fig 1: likelihood estimation during Nomalization

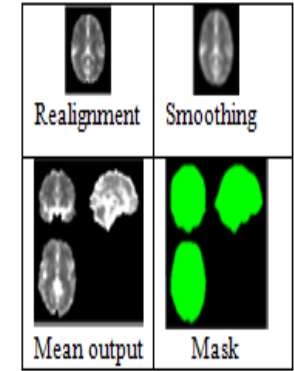

Fig 2: Preprocessing using SPM
The preprocessed outputs, Realignment, smoothing and the 3 axes mean output along with the sagittal, axial and coronal masks are shown in Fig 2. The 400 iterations of likelihood estimation of normalization are shown in Fig 1. After preprocessing using statistical parametric mapping, the seventy independent components are obtained by giving epsilon value 0.00001 for 1000 iterations. Standard PCA and fast ICA algorithms are applied .The image values are converted to $\mathrm{Z}$ score and marked on sagittal, axial and coronal view. Time point plot of 261 acquisitions of fMRI data, specifically at voxel $(32,9,20)$ and ICA time points with outlier are depicted in Fig 3. The Independent component number 52 showing outliers is one among seventy components clustered, which is inspected by experts need to be automated for further classification analysis.

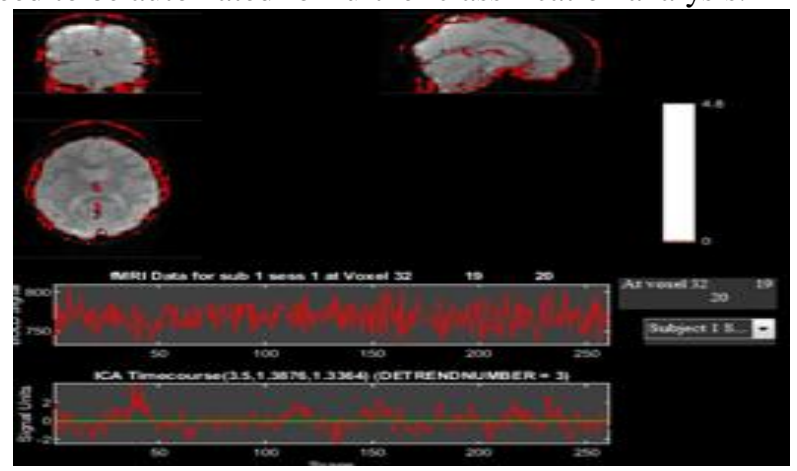

Fig 3: Component number 52 showing outlier

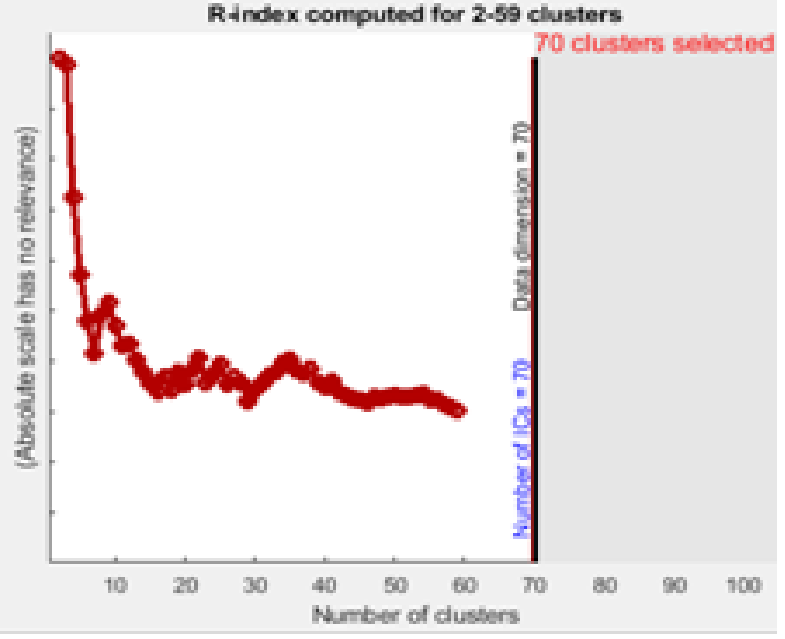

Fig 4: 70 cluster formation in ICA

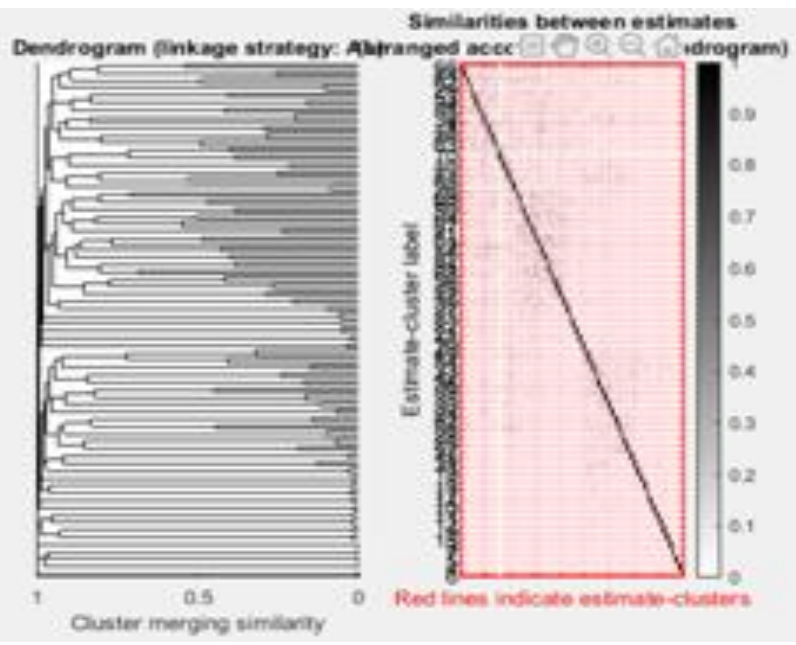

Fig 5: Dendogram and similarity

Estimate space as a $20 \mathrm{cCA}$ projection

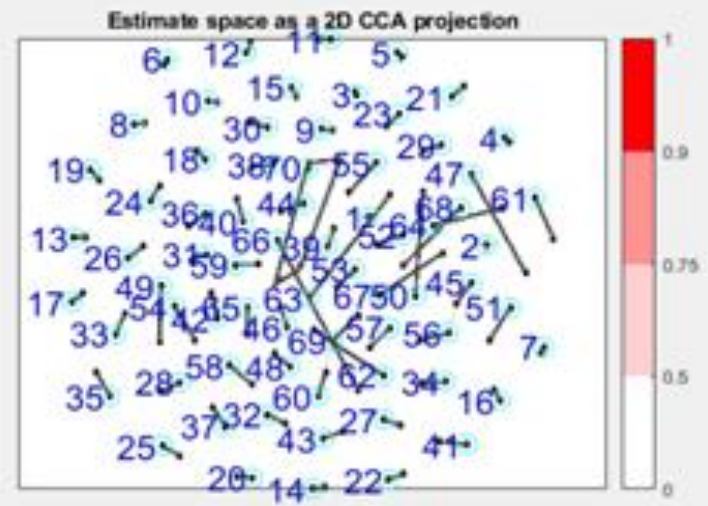

Fig 6: CCA projection of 70 clusters

During the cluster formation, the number of clusters and the corresponding R-Index are shown in Fig 4. The clusters formation build as a dendogram tree diagram and the similarity between the estimates are revealed in Fig 5. The estimation of 70 clusters are projected as a two dimensional cross correlation analysis in Fig 6 . The raw rest fMRI acquisition of both healthy and ADHD controls[6], before preprocessing shows no activation of specific regions either in the expected regions or in common networks as in Fig 7. Mostly Default Mode Network(DMN) is expected to be activated in a rest fMRI during still state or no task acquisition. The visual medial network is highly activated for ADHD Controls[13][14][15] which is depicted after SPM preprocessing and independent component analysis in Fig 8. Few voxels activated in cerebellum also coincides with existing findings.

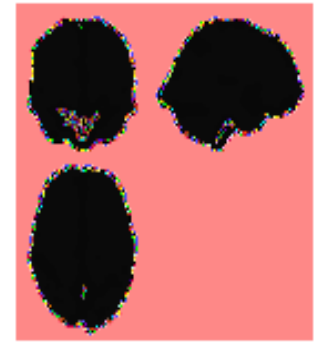

Fig 7: Raw fMRI Viewing

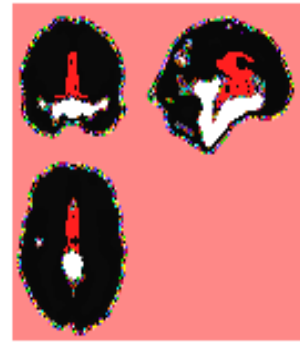

Fig 8: ADHD Activation region

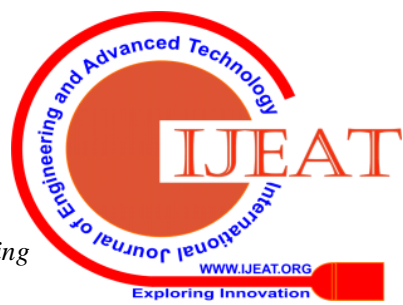


The red marker in Fig: 8 shows the middle region of all the three axes. The white regions depict the cluster of activated voxels in visual medial network. The sagittal, axial and coronal view of the visual medial network known from ground truth[11] shown can be further utilized for automatic classification of healthy and ADHD controls for Computer aided diagnosis. fMRI has progressed cognitive neuroscience investigation. Over the last twenty years, fMRI has restructured cognitive Neuroscience. In next twenty years, fMRI in cognitive neuroscience include expanded strategic precision, an expanding emphasis on network connectivity and pattern investigation, a greater attention on selective conjecture mastered by open databases, and intensified need of ontologies and computational prototypes to narrate concealed processes.

\section{DISCUSSION AND CONCLUSION}

The brain imaging has drawn special attention of distinctive neural networks in the resting state with no task, impulsive low frequency neuronal vibrations are displayed by brain. These are incidentally measured with rs-fMRI imaging and Fuctional Connective Networks is implied as the spatiotemporal correlations of signals. These allowed the non-invasive exploration of enormous dispersed functional networks in neurosurgical circumstances. Low frequency less than $0.1 \mathrm{~Hz}$ BOLD fluctuations show strong association at rest. It is concluded that the data-driven method provides an intentional structure to recognize and distinguish between individual BOLD response signals. The activated voxels is pronounced in Visual medial network of rest fMRI acquisition of ADHD controls .After hierarchical organization of the voxel correlations next to cluster analysis, the succeeding technique depends on highlights distinguished through statistical approach to time courses of group independent component analysis, which may be extracted from fMRI information. This may be exclusively automatic, unsupervised and dissimilar to different methodologies, using temporal and spatial information.

\section{REFERENCES}

1. Calhoun, VD, Adali, T, Stevens, MC, Heil, KA \& Pekar, JJ ,Semi Blind ICA of fMRI: A Method of Utilizing Hypothesis Derived Time Courses in a Spatial ICA Analysisee, NeuroImage, vol. 25,pp. 527-538,2005.

2. Amaro, E \& Barker, GJ , 'Study design in fMRI: basic principles', Brain and Cognition, vol. 60, no. 3, pp. 220232,2006.

3. Greicius, MD, Flores, BH, Menon, V, Glover, GH, Solvason, HB, Kenna, H, Reiss, AL \& Schatzberg, AF, 'Resting-state functional connectivity in major depression: abnormally increased contributions from subgenual cingulate cortex and thalamus', Biologicalhiatry, vol. 62, no. 5, pp. 429-437, 2007.

4. Buckner, RL, Andrews-Hanna, JR \& Schacter, DL , 'The brain's default network: anatomy, function, and relevance to disease',Annals of New York Academy, vol 1124, pp.1-38,2008.

5. Martin Lindquist, A, Ji Meng Loh, Lauren Atlas, Y \& Tor Wager, D , 'Modeling the Hemodynamic Response Function in fMRI: Efficiency, Bias, and mismodeling', Neuroimage, vol. 45, no. 1, pp. S187-S198, 2009.

6. Pyka, M, Hertog, M, Fernandez, R, Hauke, S, Heider, D, Dannlowski U \& Konrad, C 2010, 'fMRI data visualization with BrainBlend and Blender', Neuroinformatics, vol. 8, no. 1, pp. 21-31,2010.

7. Russel Poldrack, A, Jeanette Mumford, A \& Thomas Nichols, E , 'Hand book of functional MRI Data Analysis', Cambridge University Press, 2011

8. Mehdi Behroozi \& Mohammad Reza Daliri , 'Software Tools for the Analysis of Functional Magnetic Resonance Imaging', Basic Clinical and Neuroscience, vol. 3, no. 5.pp.71-83,2012

9. Thaiyalnayaki, SP \& Uma Maheswari, O , 'MLE-Wald and Sparse Dictionary K-SVD Learning for fMRI Post processing Analysis', in International Journal of Knowledge Engineering and Research. ISSN 2319832X. vol. II , issue II , pp. 183-187,2013.

10. Stefan Lang, Niall Duncan \& Georg Northoff, 'RestingState Functional Magnetic Resonance Imaging: Review of Neurosurgical applications', Neurosurgery, vol. 74, no. 5 , pp. $453-465,2014$

11. Emily Finn, S, Xilin Shen, Dustin Scheinost, Monica D Rosenberg, Jessica Huang, Marvin M Chun, Xenophon Papademetris \& Todd Constable, R , 'Functional connectome fingerprinting: identifying individuals using patterns of brain connectivity', Nature Neuroscience vol. 18, pp. 1664-1671,2015.

12. Vince Calhoun, D \& Tülay Adalı, 'Time-Varying Brain Connectivity in fMRI Data: Whole brain data-driven approaches for Capturing and characterizing dynamic states', IEEE signal Processing Magazine, vol.33, no.3 , pp.52-66,2016.

13. Mostert JC, Shumskaya E, Mennes M, Onnink AM, Hoogman M, Kan CC, Arias Vasquez A, Buitelaar J, Franke B, Norris DG, 'Characterising resting-state functional connectivity in a large sample of adults with ADHD'. Prog Neuropsycho pharmacol Biol Psychiatry . issue 67, pp.82-91,2016.

14. Hirbaye Mokona Lola, Habte Belete, Abebaw Gebeyehu, Aemro Zerihun,Solomon Yimer, and Kassech Leta, Attention Deficit Hyperactivity Disorder (ADHD) among Children Aged 6 to 17 Years Old Living in Girja District, Rural Ethiopia', Behavioural Neurology 1753580 , pp. 1-8,2019.

15. Anderson dos Santos Siqueira, Claudinei Eduardo Biazoli Junior, William Edgar Comfort, Luis Augusto Rohde and João Ricardo Sato, 'Abnormal Functional Resting-State Networks in ADHD: Graph Theory and Pattern Recognition Analysis of fMRI Data', BioMed Research International, 380531,pp. 1-10,2014. 